\title{
Evolution of Serious Games to Support Lifelong Learning and Decision Making: Design and Implementation of a Financial Literacy Game
}

\author{
Aldrich Rasco \\ University of Auckland \\ a.rasco@auckland.ac.nz \\ Gabrielle Peko \\ University of Auckland \\ g.peko@auckland.ac.nz
}

\author{
Johnny Chan \\ University of Auckland \\ jh.chan@auckland.ac.nz \\ David Sundaram \\ University of Auckland \\ d.sundaram@auckland.ac.nz
}

\begin{abstract}
Considering the economic changes of recent times, financial literacy arises as a focal point of interest. COVID-19, coupled with the culmination of other societal issues, underlines the importance of understanding sensible personal finance. Nationwide lockdown and other economic constraints put us in immobilised positions to confide in safe and accessible entertainment havens such as games. Herein lies an interesting research opportunity to progress personal wellbeing and capability despite the extant issues of recent times. The paper demonstrates the design and implementation of an evolving serious game that supports lifelong learning and decision making relating to personal finance. The example is a useful account of serious games' evolutionary potential to incrementally support users through lifelong learning. The game's holistic design incorporates autonomy, motivation, and support structures to ensure that lifelong learning and decision making is effectively managed through an evolving system. The corresponding implementation evidences the sheer potential of serious games.
\end{abstract}

\section{Introduction}

Research shows that autonomy, motivation, and support structure are proactive enablers of lifelong learning and informed decision making [10, 11, 14]. Serious games, therefore, as effective bearers of these attributes, are suitable for supporting lifelong learning and decision making. This paper exemplifies an evolutionary perspective in the design and implementation of serious games. The research allows for a co-created evolution between the player and the gaming environment. Financial literacy is as important as ever as we face numerous global crises such as COVID-19. The paper is further motivated to ensure financial preparation is sought from the perspective of proactive interest and future planning, as opposed to financial preparation, becoming an afterthought.

\section{Literature Review}

Nash [14] argues the importance of adopting three means to enable lifelong learning and decision making. These arguments fundamentally corroborate our claims that serious games are suitable enablers. The first argument explores the mistake in matriculating learners into fixed curricula, which understates their individual freedom to learn. Allowing the mind a certain liberty; to quest for truth promotes the imperative of lifelong learning. From our standpoint, a person's choice in games can be reflective of such an imperative. While knowledge or learning could be inconsequential to a person's reasons for playing a game, the liberty in the choice of games is clear and conducive to supporting lifelong learning. Upon enforcing one's liberty to engage with a game, the user opens oneself to constructing knowledge without the restraints of outside curricula. Further, serious games can be primed for lifelong learning, the more freedom the player has in the game [1]. This is especially paramount in large open world situations where knowledge construction can be personal y constructed and experienced [12].

Nash's [14] second argument relates to the need to motivate learners into continued learning. A metaanalysis has been conducted to study the role of serious games as a proponent of motivation [23]. Past research hypothesised a great motivational appeal and effect in serious games due to its high entertainment value $[4,7$, $11,13,23,24]$. However, the meta-analysis suggests that the motivational advantage of serious games over conventional instructional methods are not significantly higher. Many of the hypothesised reasons for this slight advantage was attributed to the role of self-determination in serious games [18, 21]. Autonomy supports the intrinsic motivation to learn, which is not always present in more instructive methods such as written tests. Interestingly, these findings corroborate Nash's [14] initial premise 
regarding lifelong learning. Successful periods of learning can be conceptualised as highly personal, subjective, and self-determined. While the high entertainment value of serious games may not be that much more motivating than instructional tasks, this does not mean that its advantage in terms of entertainment value is pointless. In fact, we argue, based on the hypotheses, that the entertainment value of serious games affirms the person's autonomous approach to learning and decision making. Essentially, the overall entertainment value of a game relies on a person's subjective evaluation of the game experience [3]. If the person so chooses to play a perceived 'highly entertaining' game based on their own liberty they will be independently motivated to play, which immediately exercises their freedom of choice at the very onset [4]. Games therefore will be immediately impactful as their corresponding user base is preemptively interested to engage with the game mechanics.

Nash's [14] third argument underlines a requirement to be able to manage lifelong learners for life. While it is not practical nor sustainable to uphold formal lifelong supporting systems, Nash's emphasises the importance of ensuring that the philosophical approach is correct. He argues lifelong learning and decision making can be empowered through maximizing our exposure to substantive learning encounters. From our view, rather than configuring traditional educators like college or university, which require great time and financial commitments; it is best to exercise the fervour for lifelong learning in more compact and universally accessible programmes [10]. In such a case, serious games appear to be a suitable vehicle. Serious games can exemplify several learning outcomes relating to behavioural change, knowledge acquisition, motor skills, perception and cognition, physiological states, social and soft skills, affective and motivational results and other niche topics $[3,5]$. The configurative potential of serious games has the capability to maximise its substantive learning encounters, in affordable and accessible means [17, 22]. To achieve this, it is important that a relevant methodology is used so that the lifelong support for learning and decision making can be conceptualised appropriately.

\section{Evolutionary Action Design Science Methodology}

Taking inspiration from action research [19] and action design science [15], the applied methodology is specifically tailored for this research. Evolutionary action design science in Error! Reference source not found. combines principles from action design and refocuses its cycles into three major iterations that aim to evolve over time. The three major iterative phases, featured in Figure 1, are development, learning and evolution. Each cycle goes through the following steps subsequently: problem formulation, design-buildevaluate, reflection and learning, and formalisation of learning. The framework has been specifically adapted for this research by including the element of intervention in the learning and evolution cycles. This inclusion is fundamental in evaluating the artefacts later in the study.

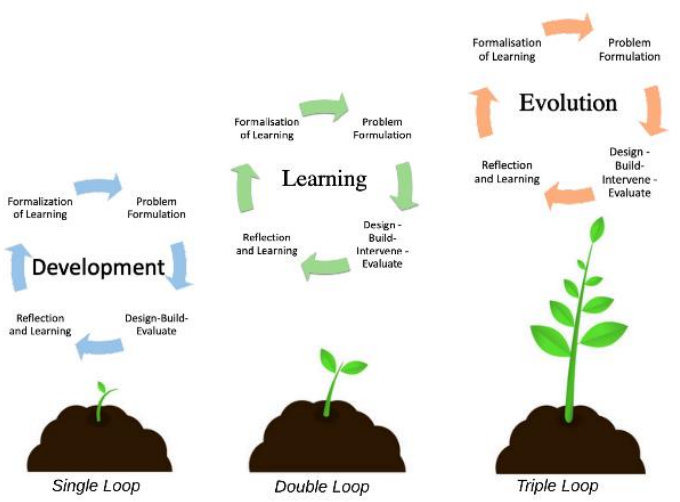

Figure 1. Evolutionary Action Design Science Framework. Adapted from [15]

Each of these phases subsequently portray the levels of learning. Single loop level of learning in the development phase encourages the developer to create several artefacts that conceptualises the situation. In the case of this research, several games have been created to reflect the problems of youth financial literacy. In the learning phase, the initial artefacts created are matured through experienced use and exposure to the artefacts and the environment. The cycle's title of learning is associated with the users' enhanced knowledge of the situation after several raw iterations in the development phase. The evolution phase finally aims to evolve the works from previous phases to implement a co-evolving situation between the artefacts, the user, and the environment. In the case of this research, long term user evolution will be attempted while having a co-evolving game to support the users' growth. The following conceptual framework in the following sections will expose how the evolving methodology will be materialised into an evolving serious game.

\section{Conceptual Framework}

The conceptual framework featured in Figure 2 is adapted from the IPP framework with an expansion on the evolutionary component [16]. The framework 
evokes an aspect of immersion, persuasion, personalization, and evolution. The crux of the framework is that as the user delves deeper into the game situation, the user goes further with the four aspects. Essentially, the user's real-life characteristics is compared in parallel to their in-game character. As the user improves in the game, the corresponding growth can be actualised in real life.

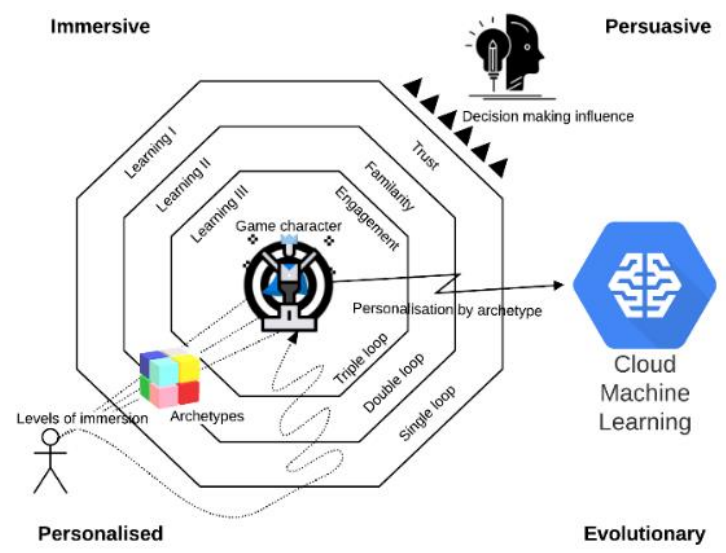

Figure 2. IPPE Framework. Adapted from [16]

The game experience should also reflect elements of single loop, double loop and triple loop learning as the user continues to play the game [2, 20]. For example, at the level of single loop of learning, error correction can be applied in tasks in the game. Mistakes can be corrected by recognising the in-game situation through trial-and-error and repetition. At the level of double loop learning, the user can realise the right values to successfully complete the game. For example, patience may be important during a maze component of the game. So, an understanding of this concept may lead to successful revisions of strategy and the underlying assumption. At the level of triple loop learning, conceptualisation of the overall game context and purpose can be achieved. In these cases, the thematic approach to the game will be understood. When users start to comprehend the learning element in the serious game, the user is better able to exercise the liberty to explore the in-game dynamics to maximise the learning potential of the game.

\section{Design of Evolutionary Serious Games}

To realise the conceptual framework of evolving serious games (Figure 2), several prototypes have been created as part of the overall research. However, this paper will only focus on the latest prototype, which is a game called Debt Maze. The purpose of the game is to enhance the user's financial knowledge regarding financial debts.
Fundamentally, the function of evolution manifests for both the user, as well as the gaming platform. While evolution occurs primarily for the user, the game adjusts itself to maintain a growing experience for the user. To get a good grasp of this idea, it is important to define the different loops of learning that subjects the user's, and the game's, coevolution [2]. At the level of single loop learning, the user is set at the rudimentary level of error-correction. The user will have to follow the correct paths in the maze, as well as provide correct solutions to progress. At the level of double loop learning, the user is set to think about the effectiveness of decisions, whilst recognizing different pathways to completion. For example, choosing a harder path over easier paths will be evaluated against each other, and the effectiveness of taking either will be kept in mind. For instance, a more challenging path may lead to more game points and skill progressions however it will dramatically challenge the player's ability. On the other hand, an easier path may harness less rewards despite being more beginner friendly. At the triple loop learning level, conceptualisation of the game crossovers with the user's innate learning outlook, which informs their strategic and operational approach in the game. In the same example, users may eventually realise that given the purpose of the game is to improve financial understanding, it may encourage them to take harder paths as opposed to easier paths. This is because the harder paths may expose the user to more pitfalls regarding personal finance and thus allow the maximisation of the user's learning. The exact specifics of the game' evolutionary design will be explained in the following section.

\subsection{Game Setting}

The player character starts in a maze with various obstacles and traps that relate to financial concepts. There are different types of routes that will be available depending on the user's financial knowledge. Reaching the house at the end of the maze, on time, is the goal of each level. Upon completing the maze, the player character becomes the legal owner of the house. That is, if the player character has not been evicted yet by the completion time constraint of the game.

Using the adapted IPPE framework (Figure 2), the game is set so that the player character's growth is immediately relatable to the user. Through characterising the nature of financial debt like a debt maze, the first instance of learning is presented to the user. This idea is then further augmented by populating the debt maze with other familiar financial concepts, such as paying off overdue rent. 


\subsection{Play Experience}

The play experience focuses on imagining the player as potential house owners. The burden of housing debt is immediately put into perspective by portraying its complexities akin to a maze with various traps. The player is tasked with familiar real-life concepts such as paying fees and maintaining fortnightly mortgage payments on time. If the player fails to do such tasks, the credit rating of the player lowers. If the credit rating reaches zero, the player is evicted from the maze and forfeits the mortgage. The goal of the game is to maintain the house by paying the mortgage and its fees in time. All while ensuring that the financial credit score is kept at a healthy level i.e. over three stars.

\subsection{Learning Goals}

There are several learning goals in this game that differ at each level. However, the first few levels will be discussed. The first learning goal is to understand the timeliness of debt payments: If the debts are not paid in time, credit rating will decrease, which may subsequently lead to eviction (or a failed game). The second learning goal is to understand rising interest rates on bad credit ratings. On occasions where the player has accrued lower credit rating, the interest charge on the base debt, compounds its value if the payment has not been payed. This means the longer it is delayed, the player will suffer penalties in terms of payments. In terms of the maze, it will be harder to access maze passages and get bonus items from the game. All in all, having good financial knowledge will allow the player to cruise through the levels. Specific levels that require specific financial knowledge will challenge players that are unfamiliar with the learning goals in that level.

\subsection{Game Goals}

The overarching goal of the game is to repay the mortgage in time and reach the end of the maze. The four essential steps to get there are the following:

1) Navigate around the maze filled with financial misdirection.

2) Avoid traps through application of financial knowledge

3) Open doors interact with characters and finish narratives to successfully pay off the week's mortgage payment.

4) Have enough money to repay the weekly mortgage payment.

\subsection{Narrative Struggle}

At the very start of the maze, the player starts with a $\$ 500,000$ mortgage. Upon gaining money and paying off mortgage payments and other fees, the player will progress through the maze. The end allows the player to enter and claim the house.

Understanding and navigating through the maze requires excellent financial knowledge. Traps will be harder to avoid without the correct understanding of the level's niche financial concept. Furthermore, the limited completion time pressures the player to make timely informed decisions. All in all, the environment will challenge the players financial literacy on different topics for every level.

Environmental obstacles, traps, bad routes, and the time limit will need to be managed efficiently and correctly to reach the end of the maze. This draws a direct parallel to the intricacies of housing debt, which requires timely financial knowledge to avoid the traps that may increase the payments and issues with house ownership.

\subsection{Feedback Loop}

The feedback system is immediately addressed in the UI of the game. Firstly, the time limit at the top of the head-up display shows that mortgage and fees payments that are expected to pay within the time limit. Failing to do so will decrease the credit rating (out of 3 stars in the UI). Losing all three credit rating stars will evict the player from the mortgage and the game. Eviction is the first fail condition of every level.

Triggering financial traps will reduce the player's credit rating. Low credit rating scores will add a multiplicative value on the current debt principal amount. Essentially low credit rating will add interest on the $\$ 500,000$ starting mortgage debt. This means that the goal of reaching the end will be more challenging as there are more payments to be made.

This will inform the player of bad financial routes and decisions that has been taken during the maze. The maze will prompt the user with either-or scenarios to direct and distinguish good routes from bad routes. A correct answer to these prompts will self-evidently inform a good decision if a money item or a door platform is reached. Please note that money items and a door platform is necessary to advancing further in the maze. Alongside the time component, a quick reach of the mandatory money items and doors informs good player decision-making

\subsection{Game Summary}

The Debt Maze game explores the precariousness 
of housing debts from the perspective of house owners. It is complicated, confusing and requires several decision points to own the house completely and legally. It is almost like a maze, full of traps with good routes and bad routes. Good routes may lead us to early, or on-time completions of mortgage and fee payments. This is beneficial to our credit ratings and possibility of fully owning a house once the mortgage and all the fees have been settled. Alternatively, taking bad financial routes in this metaphorical maze can lead us astray. Traps exists in contentious decision points. Without the right financial knowledge, we may get lost further in this financial maze. Bad routes will make us vulnerable to credit rating decrements, and in direr situations, we may even be vulnerable to eviction once lenders like the bank lose trust in our financial situations. The game conceptualises the mortgage reality in a mazing simulation.

\subsection{Learning context}

Senses of unfamiliarity, confusion and difficulty is associated with housing debt, which is framed like a 'maze'. Without sufficient understanding of housing debts, the player would not be able to complete the maze. Through constant reflections of the decisions taken in the maze, this could be changed

The game can be a starting point of debate regarding the confusions, crossroads, and complexity of housing debt. Whereby, an excellent understanding of housing debt puts the person at a financial advantage over those who do not.

\subsection{Decision making}

Decision making in the game will be exercised by applying financial knowledge to access good routes. Good routes and decisions will also lead the player to the doors (this would allow them to pay fees and debt). Please note that accessing doors takes away from the principal amount of the debt, therefore, to reach such platform suggests that the player has taken a good financial route. Bad routes lead to more vulnerable positions or in trap-filled areas. In these areas, the credit rating can decrease very quickly. Good routes will be rewarded by giving the player bonus time to complete the maze.

\subsection{Immersion, Persuasion, Personalization and Evolution Component}

Immersion is achieved as the in-game character is characterised based on the players' own characteristics. The player will have to take a quiz at the start where they are given a specific archetype. This archetype will then enhance the corresponding in-game character's skill points, which will help them complete the game.

Persuasion is achieved as the player encounters real life concepts within the game. Ideas such as the mortgage debt crisis will be reflected by giving the player character thematic tasks. For example, the player character will be tasked to pay mortgage debt payments weekly in the game.

Personalization is achieved as the player is given personalized archetypes to play the game. Players who score highly in the initial quiz will be rewarded with a strong in-game character with beneficial skills.

Evolution is achieved in the game as the player grows alongside their in-game character. The more successful tasks the in-game character achieves in the game, the more skilful and knowledgeable it becomes. Evolution for the in-game character and the player occurs as the difficulty level is adjusted based on the ease with which the player is completing the levels. Evolution occurs through scaling the games features, for example, the completion time limit will be decreased for the more financially equipped player. Evolution is also manifested by learning analytics in the maze. The credit rating score accumulated by the player will pose distinct challenges at different skill levels. The system will therefore adapt to the player and will be incrementally harder as the player progresses and scores higher in the game.

\section{Implementation of an Evolutionary Serious Game}

To implement the evolving serious game design, the system architecture featured in Figure 3 is explicated. The user will have to register in the webserver for an account. In the webserver, the user will also take an initial quiz that will give them their financial personality archetype. The data will be stored in a MySQL database. The database will feed the webserver the account details whenever it is called from the webserver. The webserver is also connected to a cloud machine learning service that applies a classification algorithm to automatically update the player's current financial personality archetype. While players receive one at the start, progression in the game may change their personality archetype. Along with sorting the players, the classification algorithm will also allocate each player with a level of reasonable difficulty. Debt Maze is a game that runs using Unreal Engine 4.24.2. It communicates to the webserver through restful communications.

Debt Maze will be a separate game application to the webserver; however, it will maintain communication through HTTP requests. Debt Maze will take in all the data processing from the webserver 
and directly update the game situation accordingly. The platform used for the game is Unreal Engine 4.24.2 and will handle the requests through a plugin called VaRest.

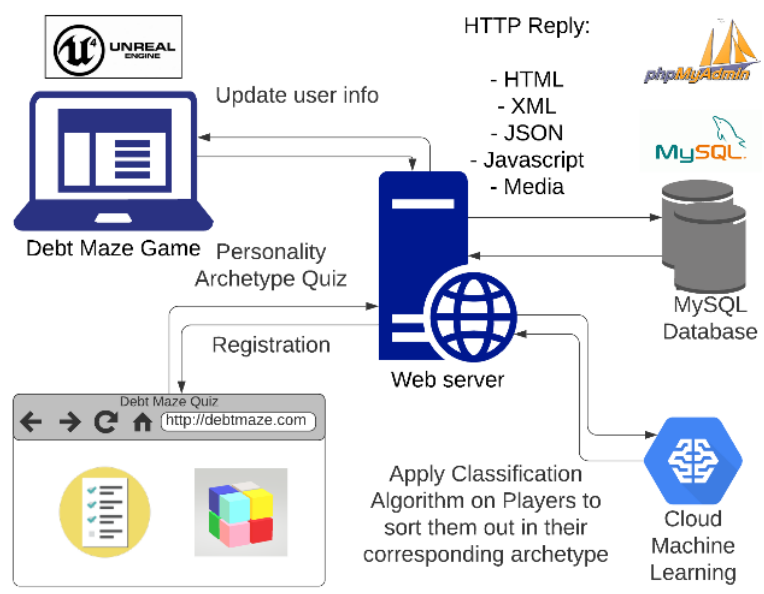

Figure 3. System architecture

\subsection{Implementation of System Architecture}

The system is hosted in a webserver through MyPHPAdmin. Figure 4 illustrates the web page. Here all communications with the MySQL database and classification learning algorithm will be handled. The user will be able to take the Debt Maze quiz here. Afterwards, the user will be awarded an archetype that will be used in the game. The user will then have to confirm their registration and once they do, their user information will be stored in the database. The Debt Maze game can then utilise the user details to login to the game.

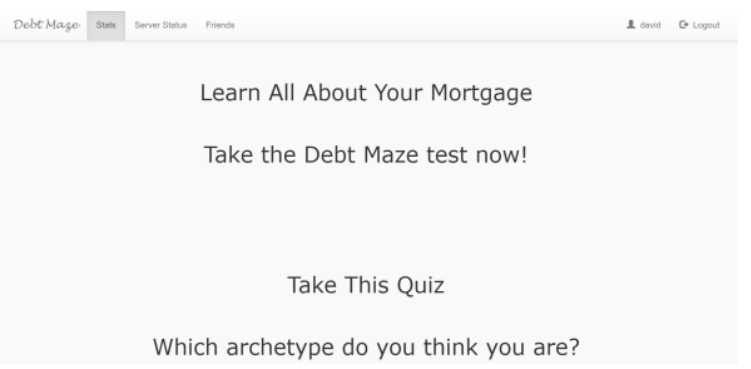

Figure 4. Web server

After the registration has been completed, the user should now open the game application via Unreal Engine. The initial bootup will prompt a login page. Here, the username and password will be asked from the user. Upon entering the details, the game application will send an HTTP request to the webserver and confirm if the user is registered. The game application will send the username and a hashed version of the password into the webserver. There, the webserver will verify with the stored database whether the details are legitimate. Once verified, the user will be taken to the main menu page. The game executes using Unreal Engine 4.24.2. While the platform does not have direct support for restful communications, we used the VaRest plugin to maintain a restful exchange between the game and the webserver. After the communication is successful, the game loads into the main menu screen.

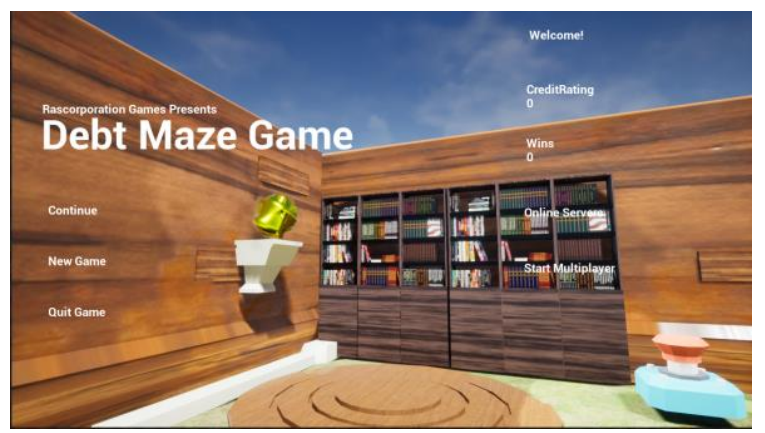

Figure 5. Main menu

Figure 5 features the Main Menu, users are formally welcomed with a personalized portal. Details such as the user's credit rating score and win total will be requested from the database. These scores will be accumulated through game progression and will be the ultimate reflection of the player's current financial literacy level. The following buttons are interactive: "Continue", "New Game", "Quit Game", "Online Servers" and "Multiplayer". The buttons' titular descriptions will bring the user to a corresponding landing page. For example, "New Game" and "Continue" will take the user to single player mode. If there is a saved file found in the game, the user can continue previous game sessions. The user is also able to play in multiplayer mode using the rightmost buttons. "Start Multiplayer" will start a game lobby, whereas "Online Servers" will browse existing lobbies.

\subsection{Scenario-based Features that Support Lifelong Learning and Decision Making}

While there are several gaming features within the game that reflect financial learning and decision making, this section will outline procedural examples that exhibit lifelong learning and decision making based on a scenario. These examples will showcase the game's support for simultaneous co-evolution between the player and the game environment.

The basic implementation of financial literacy tests in the game occurs as the player is required to answer a basic questions regarding credit cards. If the 
correct answer is selected, the player will be able to access a safe or good route. Otherwise, the wrong answer will punish the player with a vulnerable or bad route. Each type of route has its own consequences, these will be explained later.

The display interface also features several variables that will act as basic stats for the player. The three starts to the left symbolises the player's credit rating. This variable will increase or decrease based on the choices the player makes during the game. Triggering traps, following bad routes, and running out of time will negatively affect the credit rating. If the player's credit rating reaches zero stars, the player will lose. The player must reach the end with at least 1 credit rating star to finish the game. The money icon under the credit rating variable represents the player's money. This will indicate how much the player has accumulated per level. Money can be picked up from ATM machines which will be scattered throughout the level. The bank icon underneath the money icon reflects the total amount of loan payables the character is liable for. This principal amount must be paid off before the time. Once it is paid, the message at the top right corner should update to "Loan Paid Off!" Once this appears, the player can finish the level and proceed to the next. The credit score number represents the overall credit score of the player. This will be updated regularly in the game as the player finishes a level. Improving the credit score will give the player in-game benefits that will help them finish the maze successfully.

Figure 6 features the basic dynamic with ATM machines. The player can withdraw different amounts of money with different ATMs. Good routes will often reward the player with ATMs. This practice is reflective of the advantage of financial knowledge. A metaphor is drawn out as players are rewarded financially by making the right choices.

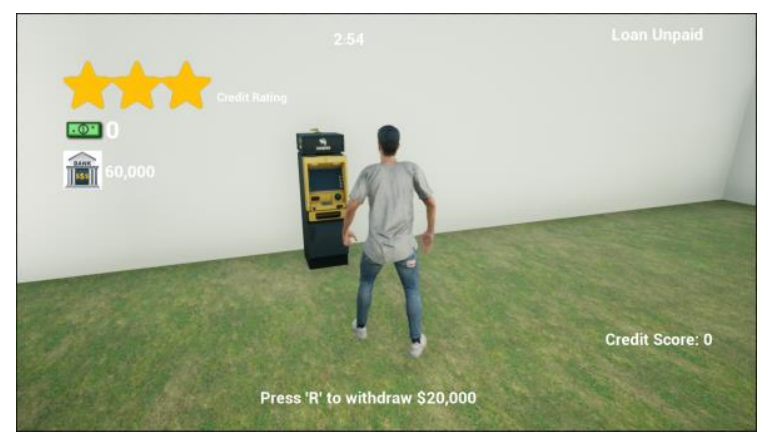

Figure 6. ATM interaction

Figure 7 features the traps that are present in the game. Bad routes are areas populated with traps that will decrease the player's credit rating. Once the credit rating hits zero the player will lose the game. It is therefore the player's mission to maintain at least one credit rating star before ending the game. It will also be advantageous to avoid traps and do well in the financial tests that the game enforces onto the player. While entering bad routes may be disadvantageous to the player's progress, the game will also feature catch up mechanisms to allow players to learn from their mistakes. Players will usually be able to recover from bad decision making by solving puzzles in trapped areas. These puzzles will not only expose the player to common financial pitfalls, but it will directly reflect the opportunistic nature of financial recovery. While the game appears to be largely metaphorical in its learning approach, real life anecdotes will manifest in catchup mechanics to imitate real life problems. For example, a player stuck in a bad route will encounter simple budgeting tasks to put them back on track towards good routes.

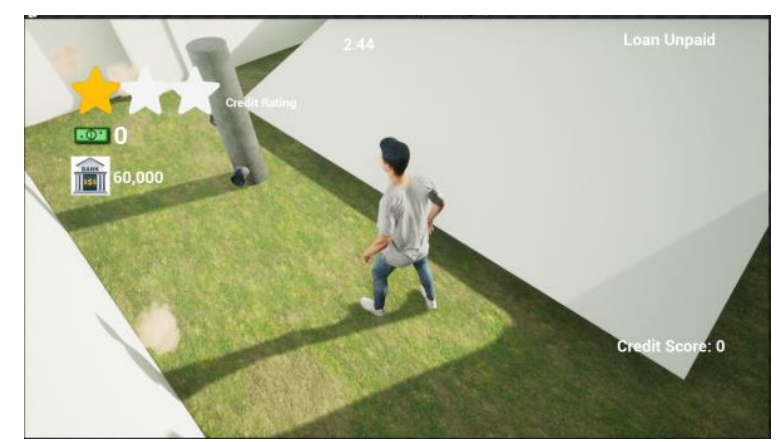

Figure 7. Traps in bad routes

Mortgage repayment is central to the game's theme. The dynamic featured in Figure 8 requires the player to pay off a part of the debt to the banker. Doing so will reduce the principal amount of the user's debt.

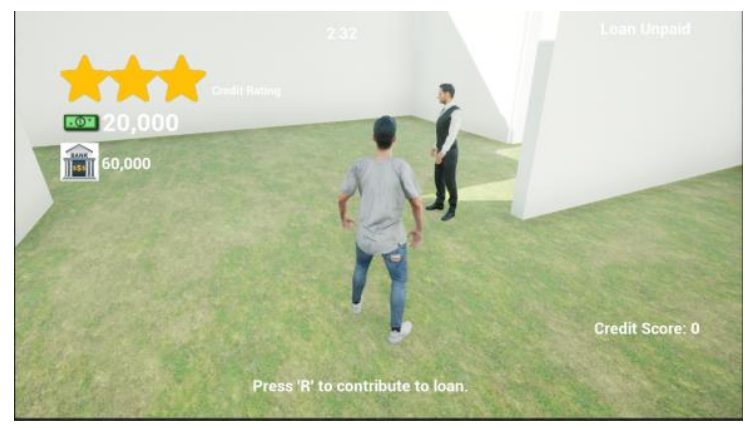

Figure 8. Mortgage payment

Figure 9 features the end game dynamic of each level. Once the total amount of the loan has been paid off the player can then complete the game. The player would need to find the house. Inside there is a white circle that will take the player to the next level. If all the loan has been paid off and the player has at least 
one credit rating star, the player will be sent to the next level.

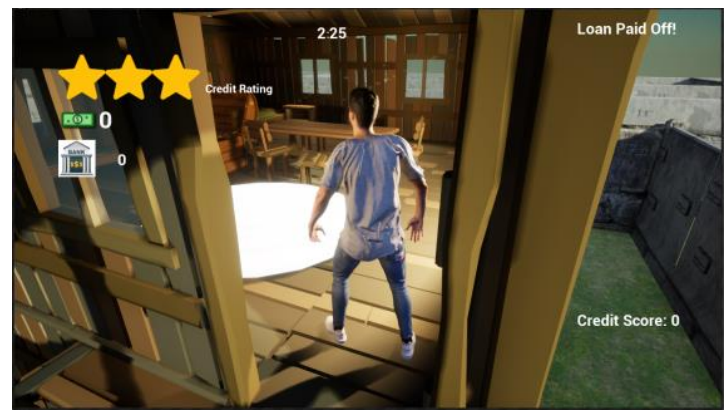

Figure 9. Completing levels

Figure 10 features the benefits of having high credit scores. Throughout the game skill upgrades are available to the player depending on their credit score. In the scenario above, the player has a credit score of 8000. This makes the player eligible for additional completion time, movement speed buffs, jump velocity buffs, credit rating replenishments and cash bonuses. These upgrades will help the player complete the game faster and more efficiently. These skill upgrades come easily when correct financial knowledge is applied in the events presented in the game.

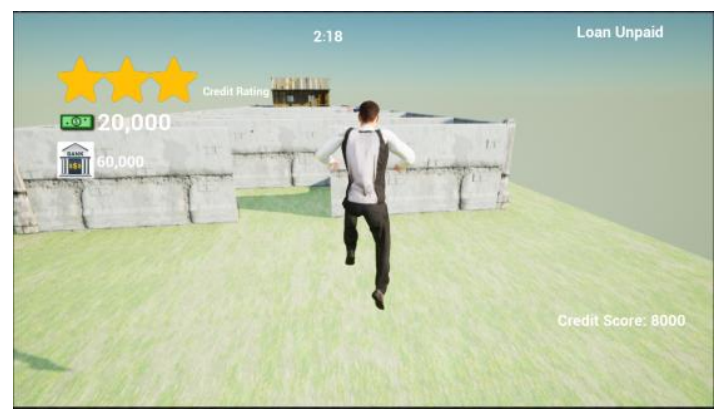

Figure 10. Skill upgrades

Figure 11 features the possibility of multiplayer in the game. While co-evolution occurs predominantly between the user and the game environment, some aspects of evolution are gained through learning from other players. It works similarly with the predominant type of co-evolution; users reach higher levels of learning upon observation and eventual manifestation of triple loop learning.

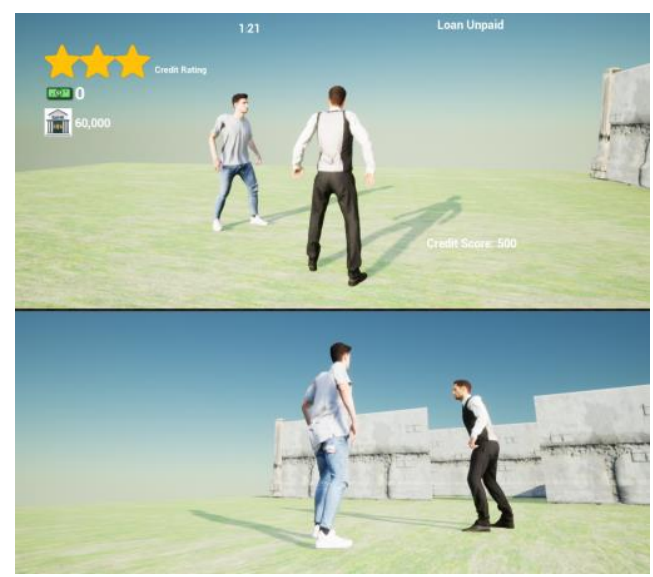

Figure 11. Multiplayer component

\subsection{Evaluation}

Our research is continually evaluated with guidance from design science evaluation guidelines [8]. The ways with which the study has been evaluated is further explained below.

6.4.1. Descriptive analysis. We have evaluated the utility of Debt Maze through cross referencing its features with the Dreyfus model of skill acquisition [6]. The crux of the research is to be able to take a novice in personal finance into competent financial decision makers. Debt Maze supports novices, advance beginners, competent players and proficient players [6]. Novices are supported on the very onset, as instructions are directly tasked through prompts throughout the game. For example, the player is asked to 'pay off your loan' instructively through character interactions. Advanced beginners are also manifested as the player is situated in less instructed situations. The open world feel of the game allows for dynamisms in sandbox-like parameters. This allows the advanced beginner to identify new aspects and maxims outside direct instructions. As players enter latter levels in Debt Maze, a sense of what is important starts to arise. This transition signals the game's support for competent players as more reflective and creative thinking is required to complete the levels. Players begin to restrict themselves to fewer relevant aspects of the maze. Thus, willing themselves to adapt to dynamically changing game parameters. Last, as levels become more complex, the game attempts to emotionally involve the player. Through interweaving more complex narratives and allowing the player to experiment more creatively in the sandbox world. The Debt Maze supports proficient players as well, as they are characterized to be involved freely in the game dynamics while driven by emotional involvement. The variety of open world interactions and side quests allows for this level of 
exploration which overall lifts its utility regarding personal finance improvement.

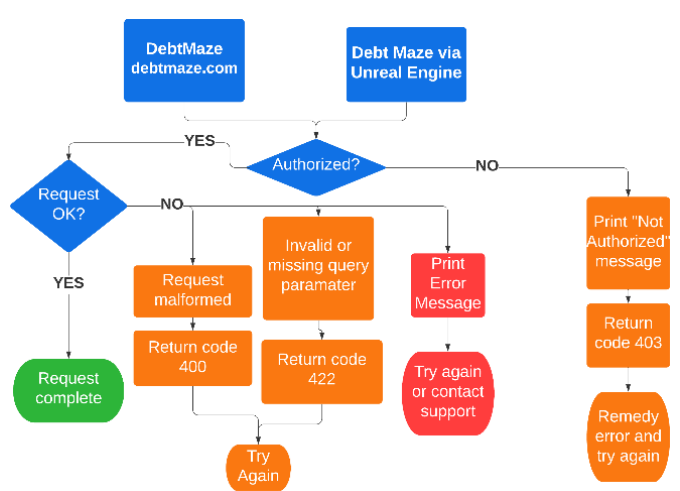

Figure 12. Evaluation score

6.4.2. Structural testing. The Debt Maze has gone through extensive white box testing to discover flaws and failures in execution paths As an example, Figure 12 outlines the testing process used to verify the HTTP communications of the game with our local host server. While there were several test cases that initially failed, the experiences have helped diagnose, and eventually fix the errors. Now, the Debt Maze still requires further testing to fully validate all features. However, at the minimum viable level, the Debt Maze operates well as a prototype for the research.

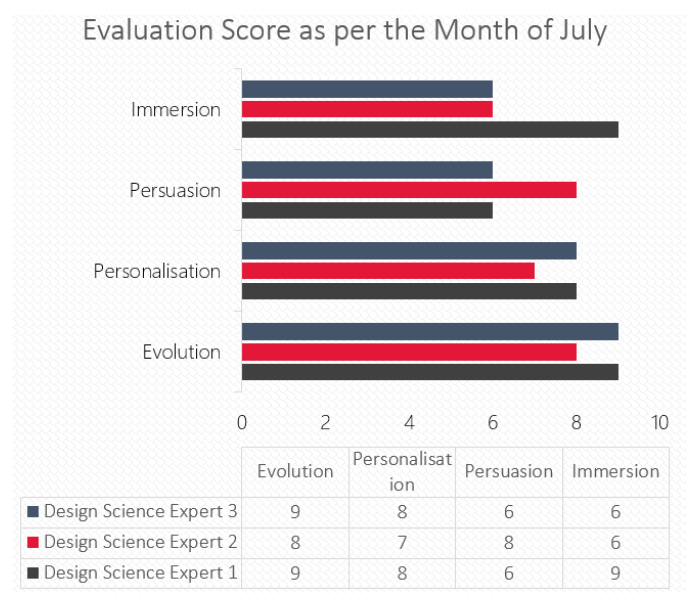

Figure 13. Request testing

6.4.3. Functional testing. The Debt Maze has also gone through extensive black box testing. As a prototype based on learning, the learning utility is especially validated in this research. Central to the frameworks used in this study are the aspects of immersion, persuasion, personalization, and evolution of the game. In direct reflection to its' relation to personal finance, these tenets are evaluated by design science experts on a monthly basis through a score. For the month of July, the game is given the subsequent scores, as featured in Figure 13. These are then reflected upon by the researchers to vastly improve the tenets in hopes of gaining a higher score in the next monthly iteration.

6.4.3. Validation through publication. The evolutionary action design science methodology used in this research has been validated through its publication by the Association for Information Systems (AIS) e-library [15]. Intrinsic to this methodology, is its evaluative nature which especially considers scholarly and expert feedback to concurrently evaluate and develop the Debt Maze. Several features that emerge from this validation sequence has originated from previous publications as well. For instance, the IPPE framework [16] was paramount in underpinning the fundamental learning framework of personal finance.

6.4.4. Scenario testing. The scenario testing has been instrumental in ensuring that the game is nevertheless still relevant to personal finance. Through testing gameplay, it is ensured that the Debt Maze incorporates key concepts money basics, investing, protecting, and borrowing. These key concepts are selected especially from Huston's account of the main components of financial literacy [9]. The vision for the research is such that the improvement of financial literacy will eventually improve people's grasp of personal finance. As such, further testing is still required in this research to fully validate the key concepts. The subsequent stage of the research is to pilot the game in financial literacy workshops held at high schools. Students at the ages of 13 to 18 years would be invited to play these games on a longitudinal basis. Through a contemporary research process that includes data collection and analysis, the research aims to evaluate the design artefacts. Overall, their progress will be consistently measured in the game will be measured against real life applications of sensible personal finance.

\section{Conclusion}

To conclude, the paper demonstrates the design and implementation of an evolving serious game within the context of financial literacy. The game Debt Maze serves as a testament to evolutionary serious game learning where evolution manifests for the player and the game. Debt Maze's holistic design, incorporates autonomy, motivation and support structures that fundamentally maintains its incremental support for lifelong learning and decision making. This then empowers the user to go through multiple learning 
levels including single loop learning, double loop learning and triple loop learning. While a personal evolution occurs for the user, the game platform is also able to adapt to the user's growth, which demonstrates the intrinsic evolutionary potential of serious games alongside the player. The importance of this contribution testifies to the potential of serious games as a viable and adaptive decision support system, that is capable of being immersive, persuasive, personalized, and evolutionary in nature.

\section{References}

[1] Abt, C.C., Serious games, University press of America, 1987.

[2] Argyris, C., "Single-loop and double-loop models in research on decision making", Administrative science quarterly, 1976, pp. 363-375.

[3] Connolly, T.M., E.A. Boyle, E. Macarthur, T. Hainey, and J.M. Boyle, "A systematic literature review of empirical evidence on computer games and serious games", Computers \& Education 59(2), 2012, pp. 661686.

[4] Dale, S., "Gamification: Making work fun, or making fun of work?", Business Information Review 31(2), 2014, pp. 82-90.

[5] Dicheva, D., C. Dichev, G. Agre, and G. Angelova, "Gamification in Education: A Systematic Mapping Study", Journal of Educational Technology \& Society 18(3), 2015, pp. 75-88.

[6] Dreyfus, S.E., and H.L. Dreyfus, A five-stage model of the mental activities involved in directed skill acquisition, California Univ Berkeley Operations Research Center, 1980.

[7] Garris, R., R. Ahlers, and J.E. Driskell, "Games, motivation, and learning: A research and practice model", Simulation \& Gaming 33(4), 2002, pp. 441467.

[8] Hevner, A., S.T. March, J. Park, and S. Ram, "Design science research in information systems", MIS quarterly 28(1), 2004, pp. 9-22.

[9] Huston, S.J., "Measuring financial literacy", Journal of Consumer Affairs 44(2), 2010, pp. 296-316.

[10] Knapper, C., and A.J. Cropley, Lifelong learning in higher education, Psychology Press, 2000.

[11] Malone, T.W., "Toward a Theory of Intrinsically Motivating Instruction*”, Cognitive Science 5(4), 1981, pp. 333-369.

[12] Merrick, K.E., and M. Lou Maher, "Motivated reinforcement learning for adaptive characters in openended simulation games", Proceedings of the international conference on Advances in computer entertainment technology, (2007), 127-134.

[13] Morschheuser, B., and J. Hamari, "The gamification of work: Lessons from crowdsourcing", Journal of Management Inquiry 28(2), 2019, pp. 145-148.

[14] Nash, D.A., "The Life-Long Learning Imperative... Ends and Means", Journal of Dental Education, 1994.

[15] Rasco, A., J. Chan, G. Peko, and D. Sundaram, “Serious Financial Games for Youth: An Evolutionary Action Design Science Approach”, 2020, pp. 1-10.

[16] Rasco, A., J. Chan, G. Peko, and D. Sundaram, "FinCraft: Immersive Personalised Persuasive Serious Games for Financial Literacy Among Young DecisionMakers", Proceedings of the 53rd Hawaii International Conference on System Sciences (2020), 1-10.

[17] Ritterfeld, U., M. Cody, and P. Vorderer, Serious games: Mechanisms and effects, Routledge, 2009.

[18] Ryan, R.M., C.S. Rigby, and A. Przybylski, "The motivational pull of video games: A self-determination theory approach", Motivation and emotion 30(4), 2006, pp. 344-360.

[19] Sein, M.K., O. Henfridsson, S. Purao, M. Rossi, and R. Lindgren, "Action design research", MIS quarterly, 2011, pp. 37-56.

[20] Tosey, P., M. Visser, and M.N.K. Saunders, "The origins and conceptualizations of 'triple-loop'learning: A critical review", Management learning 43(3), 2012, pp. 291-307.

[21] Uysal, A., and I.G. Yildirim, "Self-determination theory in digital games", In Gamer psychology and behavior. Springer, 2016, 123-135.

[22] Wendel, V., S. Göbel, and R. Steinmetz, "Seamless learning in serious games-how to improve seamless learning-content integration in serious games", International Conference on Computer Supported Education, (2011), 219-224.

[23] Wouters, P., C. Van Nimwegen, H. Van Oostendorp, and E.D. Van Der Spek, "A meta-analysis of the cognitive and motivational effects of serious games.", Journal of educational psychology 105(2), 2013, pp. 249.

[24] Wouters, P., and H. Van Oostendorp, "Overview of instructional techniques to facilitate learning and motivation of serious games", In Instructional techniques to facilitate learning and motivation of serious games. Springer, 2017, 1-16. 